\title{
Assessment of Type 2 Diabetes Patients' Knowledge of Oral Hypoglycemic Agents
}

Milica Paut Kusturica, MPharm, Ph.D. ${ }^{1}$, Mina Maričić, M.D. ${ }^{2}$, Ana Tomas Petrović, M.D. ${ }^{1}$, Tihomir Dugandžija, M.D., Ph.D., ${ }^{3,5}$ Veljko Crnobrnja, M.D., Ph.D. ${ }^{4,6}$, Olga Horvat, M.D., Ph.D. ${ }^{1}$

Department of Pharmacology and Toxicology, ${ }^{2}$ Faculty of Medicine, ${ }^{3}$ Department of Epidemiology, ${ }^{4}$ Department of Pathophysiology and Laboratory Medicine, Faculty of Medicine, University of Novi Sad, Novi Sad 21000, Vojvodina, Serbia.

${ }^{5}$ Oncology Institute of Vojvodina, Institutski put 4, Sremska Kamenica 21204, Vojvodina, Serbia.

${ }^{6}$ Center of Laboratory Medicine, Clinical Center of Vojvodina, Novi Sad 21000, Vojvodina, Serbia.

Received 15 June 2019 • Revised 10 October 2019 • Accepted 15 October 2019 • Published online 2 December 2019

\section{Abstract:}

Objective: Considering that adherence level affects diabetes treatment success and maintenance of glycemic control greatly, the aim of this study was to examine diabetes patients' adherence to oral hypoglycemic agents (OHAs) and knowledge about their mechanism of action, dosing regimen, and side effects.

Material and Methods: This cross-sectional study was conducted on a sample of 100 patients with type 2 diabetes in order to assess their knowledge of OHAs using anonymous questionnaires.

Results: Most patients had used OHAs between 2 and 5 years (61.0\%), where $78.0 \%$ were treated with metformin, and the remaining $22.0 \%$ were prescribed sulfonylurea derivatives. Besides drugs used for the treatment of type 2 diabetes, $58.0 \%$ of patients took another $1-3$ drugs daily for the treatment of other conditions. Although $75.0 \%$ achieved a score of 5-6 out of the maximum score of 8 , only $2.0 \%$ of respondents listed at least 2 side effects of the OHA they used, and none of them could explain its mechanism of action. Most of the information about OHAs was given to patients by endocrinologists $(53.0 \%)$.

Conclusion: More than half of participants considered their knowledge of OHAs insufficient. Results clearly indicate that the respondents were not sufficiently familiar with the mechanism of action and possible side effects of such medications. Information about OHAs given in written form as well as via community pharmacists would contribute to educating type 2 diabetes patients significantly.

Keywords: oral hypoglycemic agents, patients' knowledge, type 2 diabetes mellitus

Contact: Mina Maričić, M.D.

Faculty of Medicine, University of Novi Sad, Novi Sad 21000, Vojvodina, Serbia.

E-mail: minamaricic001@gmail.com

(c) 2020 JHSMR. Hosting by Prince of Songkla University. All rights reserved.

This is an open access article under the CC BY-NC-ND license

(http://www.jhsmr.org/index.php/jhsmr/about/editorialPolicies\#openAccessPolicy).
J Health Sci Med Res 2020;38(1):35-42 doi: 10.31584/jhsmr.202075 www.jhsmr.org 


\section{Introduction}

Diabetes mellitus (DM) is an important cause of mortality, morbidity, and health-system costs in the world. ${ }^{1}$ Globally, the number of people with DM has quadrupled in the past three decades, and DM is the ninth major cause of death. About 1 in 11 adults worldwide now has DM, 90.0\% of whom have type 2 diabetes. $^{2}$ Despite evidence showing that glycemic control using either oral hypoglycemic agents (OHAs) or insulin can reduce microvascular complications in type 2 DM, along with its associated morbidity and mortality, adherence to OHAs remains sub-optimal ${ }^{3}$, which may have a deleterious effect on glycemic control. The correct use of medicines has been highlighted as one of the ways in which people with diabetes can be supported in their efforts to self care. Poor adherence to medications may result from incomplete patient understanding of the importance of taking medications, or of their actions, or because of their side effects. Incorrect administration of $\mathrm{OHAs}$ in relation to food can result in reduced efficacy and an increased side-effect profile. ${ }^{4}$ One of the influencing factors regarding adherence to treatment regimens is the content and consistency of information given to patients by health professionals. ${ }^{5}$ The information provided to patients about OHAs may be insufficient or even too extensive; patients tend to recall only small proportions of it. ${ }^{6}$

Bearing in mind that adherence level affects diabetes treatment success, glycemic control maintenance, and side effect expression greatly, the aim of this study was to examine diabetes patients' adherence to OHAs as well as their knowledge about the mechanism of action, dosing regimen and side effects of these drugs. The expected benefit of this research was to guide healthcare providers in improving the education of these patients by determining existing shortcomings in their knowledge regarding these drugs.

\section{Material and Methods}

This study was conducted in apothecaries in Novi Sad, Serbia on a sample of subjects with type 2 DM from November 11, 2016 to December 28, 2016. One hundred patients with type 2 DM were interviewed during their pharmacy visit. Since type 2 DM treatment in Serbia starts with a single drug, the inclusion criterion was that subjects be on OHA monotherapy. The goal was to evaluate the knowledge of these patients regarding their $\mathrm{OHA}$ and use the findings as a guide to design educational interventions for such patients at the outset of treatment. Each respondent first read the text of the informed consent and obtained complete and detailed information about the survey from the examiner. After that, if they agreed to participate in the survey, they signed a written informed consent. Afterwards, the examiner interviewed the respondents via a questionnaire. The first part of the questionnaire referred to the respondents' demographic data. The second part referred to the respondents' knowledge about the OHA they took. Specifically, respondents were examined whether they knew the name of the OHA they took, why they took it, what effects the drug had on their body, how OHAs are used correctly in regard to time of daily food intake onset, what their possible side effects are, and what they would do when noticing a side effect or missing a dose of the drug. In addition, the participants also answered questions about whether they thought they knew enough about OHAs, how long they had taken OHAs, and whether they used other medicines on a daily basis. Point values were attached to the answers of the first 7 questions. These point values were summed up for every respondent in order to obtain a score of their OHA knowledge. Each question bore a single point, except for the second question, "Do you know why you are using this drug?", where the respondents got 1 point for saying they used it to lower their blood 
sugar level, and 2 points for describing how the drug works on the body or its mechanism of action accurately. The maximum score was 8 points. A correct answer to the question, "Do you know which are the possible side effects of this drug?", was considered the respondent knowing a minimum of 2 side effects. For patients taking metformin, common side effects like nausea, diarrhea, flatulence, myalgia, and, the rare but serious complication, lactic acidosis were accepted as correct answers. Possible answers for the side effects of sulfonylureas were considered hypoglycemia, weight gain, drug allergy, darkcolored urine, and upset stomach. The correct answer for the question "What would you do if you noticed a side effect of this drug?" was "Consult a doctor or pharmacist." When asked "What would you do if you missed a dose of this drug?", the respondents were given 1 point if they replied they had never missed a dose, or they would consult a doctor or pharmacist.

The statistical analysis was performed using the Statistical Package for the Social Sciences software (SPSS 15.0 for Windows, SPSS Inc., Chicago, IL, USA). The results are presented as frequency, percent mean, and standard deviation. The chi-square test was used to assess the differences between nominal data (frequencies). The analysis of variance and t-test were used to investigate the differences in value of knowledge scores. All p-values< 0.05 were considered statistically significant.

\section{Results}

One hundred and thirty patients were asked to participate in the study, of which 100 accepted to participate (response rate $76.9 \%$ ). About half of the participants were male, and the majority were older than 65 years and retired and had completed secondary school. The highest percentage of respondents had taken $\mathrm{OHA}$ between 2 and
5 years. Besides the drug used for the treatment of type $2 \mathrm{DM}$, more than half of the patients took another $1-3$ drugs on a daily basis to treat other illnesses, and a certain number of patients used more than 3 other drugs. Most patients were treated with metformin, while the rest were on sulfonylurea derivatives (Table 1).

Table 1 Patients' demographic data

\begin{tabular}{|c|c|c|}
\hline Variable & $\begin{array}{l}\text { Number of } \\
\text { respondents }\end{array}$ & Percentage \\
\hline \multicolumn{3}{|l|}{ Sex } \\
\hline Male & 51 & 51.0 \\
\hline Female & 49 & 49.0 \\
\hline \multicolumn{3}{|l|}{ Age (years) } \\
\hline$\leq 45$ & 4 & 4.0 \\
\hline $46-64$ & 32 & 32.0 \\
\hline$\geq 65$ & 64 & 64.0 \\
\hline \multicolumn{3}{|l|}{ Education } \\
\hline Primary school & 10 & 10.0 \\
\hline Secondary school & 66 & 66.0 \\
\hline University degree & 24 & 24.0 \\
\hline \multicolumn{3}{|l|}{ Occupation } \\
\hline Employed & 25 & 25.0 \\
\hline Unemployed & 6 & 6.0 \\
\hline Retired & 69 & 69.0 \\
\hline \multicolumn{3}{|l|}{ Oral hypoglycemic agent } \\
\hline Metformin & 78 & 78.0 \\
\hline Sulfonylurea derivates & 22 & 22.0 \\
\hline \multicolumn{3}{|l|}{ Treatment duration (years) } \\
\hline $2-5$ & 61 & 61 \\
\hline $6-10$ & 29 & 29 \\
\hline$>10$ & 10 & 10 \\
\hline \multicolumn{3}{|c|}{ Number of drugs used besides } \\
\hline \multicolumn{3}{|c|}{$\mathrm{OHA}$} \\
\hline 0 & 24 & 24.0 \\
\hline 1 & 17 & 17.0 \\
\hline 2 & 27 & 27.0 \\
\hline 3 & 14 & 14.0 \\
\hline$>3$ & 18 & 18.0 \\
\hline Total & 100 & 100.0 \\
\hline
\end{tabular}

$\mathrm{OHA}=$ oral hypoglycemic agent 
Table 2 Questions included in patients' score of knowledge on oral hypoglycemic agents and contribution of correct answers

\begin{tabular}{lll}
\hline Questions included in patients' score of knowledge & C T $^{*}$ & Percentage \\
\hline 1. Do you know the name of the drug you use to lower your blood glucose level? & $100 / 100$ \\
2. Do you know why you are taking this drug? & $93 / 100$ & 100.0 \\
a. Treatment indication & $0 / 100$ & 93.0 \\
b. Mechanism of action & $88 / 100$ & 0.0 \\
3. Do you know how to take this drug in regard to the time of food intake onset? & $99 / 100$ & 88.0 \\
4. Do you know at what time of day and how many times a day you should take this drug? & $2 / 100$ & 2.0 \\
5. Do you know the possible side effects of this drug? & $70 / 100$ & 70.0 \\
6. What would you do if you noticed a side effect of this drug? & $62 / 100$ & 62.0 \\
7. What would you do if you missed a dose of this drug? & \\
\hline
\end{tabular}

${ }^{*} \mathrm{C} / \mathrm{T}=$ number of correct answers/ total number of answers

Table 2 shows the questions whose responses were included to determine the score of the patient's knowledge about OHAs as well as the percentage of correct answers to those questions. All patients knew the name of the OHA they used. Most of them knew the indication for taking the $\mathrm{OHA}$, but none of them could explain its mechanism of action. Also, the vast majority knew how to use the drug in regard to time of food intake onset (88.0\%), while almost all of them (99.0\%) knew how many times a day they should take it. Only 2 respondents listed at least 2 side effects of the OHA they used, while one third of the respondents did not know what to do if they noticed a side effect (Table 2).

Table 3 shows the scores of the patients' knowledge about OHAs. Most of the respondents achieved a score of 5-6 out of a maximum of 8 . There were no statistical differences in the score value in relation to demographic characteristics, duration of OHA usage, and the concomitant use of other drugs ( $p$-value $=0.05$ ).
Questions not included in the calculation of the score of the patients' knowledge about OHAs, as well as their answers, are presented in Table 4. About one third of the respondents said that they did not know what they would do if they missed taking their medication. Approximately half of the respondents opined that they knew enough about their $\mathrm{OHA}$ and its possible side effects. Most of the information the patients had about $\mathrm{OHAs}$ was obtained from endocrinologists.

Table 3 Score of patients' knowledge about oral hypoglycemic agents

\begin{tabular}{lll}
\hline $\begin{array}{l}\text { Score of } \\
\text { knowledge }\end{array}$ & $\begin{array}{l}\text { Number of } \\
\text { respondents }\end{array}$ & Percentage \\
\hline $0-2$ & 0 & 0.0 \\
$3-4$ & 24 & 24.0 \\
$5-6$ & 75 & 75.0 \\
$7-8$ & 1 & 1.0 \\
\hline Total & 100 & 100.0 \\
\hline
\end{tabular}


Table 4 Questions not included in patients' score of knowledge on oral hypoglycemic agents

\begin{tabular}{|c|c|c|}
\hline Questions & $\begin{array}{l}\text { Number of } \\
\text { respondents }\end{array}$ & Percentage \\
\hline \multicolumn{3}{|c|}{ What would you do if you noticed a side effect of this drug? } \\
\hline I do not know & 27 & 27.0 \\
\hline I would eat something sweet & 7 & 7.0 \\
\hline I would stop taking the drug & 9 & 9.0 \\
\hline I would consult an MD & 56 & 56.0 \\
\hline I would consult a pharmacist & 1 & 1.0 \\
\hline \multicolumn{3}{|c|}{ What would you do if you missed a dose of this drug? } \\
\hline I would take the next dose as prescribed & 55 & 55.0 \\
\hline I do not know & 38 & 38.0 \\
\hline I never have never missed a dose & 7 & 7.0 \\
\hline \multicolumn{3}{|c|}{ Do you think you know enough about the drug you use and its possible side effects? } \\
\hline Yes & 53 & 53.0 \\
\hline No & 47 & 47.0 \\
\hline \multicolumn{3}{|c|}{ From whom did you receive most of the information you know about this medicine? } \\
\hline Pharmacist & 1 & 1.0 \\
\hline General practitioner & 41 & 41.0 \\
\hline Endocrinologist & 53 & 53.0 \\
\hline Friends/family & 1 & 1.0 \\
\hline Other & 4 & 4.0 \\
\hline Total & 100 & 100.0 \\
\hline
\end{tabular}

$\mathrm{MD}=$ doctor of medicine

\section{Discussion}

According to the literature review, to the authors' knowledge, this is the first study conducted in Serbia that examines the knowledge about OHAs in patients with type 2 DM. Despite the fact that the patients achieved good scores in terms of their knowledge about OHA $(75.0 \%$ of respondents achieved a score of $5-6$ out of 8 ), only $51.0 \%$ of them answered affirmatively when asked if they thought they knew enough about the OHA they used and its side effects.

Our results show that this patient population with type 2 DM had only basic knowledge about the effects of OHAs on their body and illness. Adequate knowledge about these drugs' mechanism of action would certainly contribute to a better understanding of the correct administration of OHAs in regard to food intake and the possibility of side effects, which were identified as shortcomings regarding OHA knowledge among our respondents. While $99.0 \%$ of patients claimed to know at what time of day and how many times a day the drug should be taken, $12.0 \%$ of them said that they do not know how the OHA should be taken in regard to food intake. In other words, they lacked the knowledge that metformin should be taken with or after the meal in order to minimize gastrointestinal side effects, while sulfonylurea derivatives should be taken before the meal to avoid hypoglycemia. Educating patients about the correct administration of $\mathrm{OHAs}$ in regard to food intake would enhance treatment efficacy and reduce the risk 
of side effects ${ }^{6}$, and consequently, decrease additional financial burden on the healthcare system. ${ }^{7}$ Furthermore, it was discovered that $98.0 \%$ of respondents failed to particularize at least two possible side effects of their $\mathrm{OHA}$, which is not surprising, bearing in mind that none of them was able to explain its basic mechanism of action. Only 2.0\% particularized nausea and diarrhea as side effects, but none of was aware that sulfonylurea derivatives could lead to hypoglycemia or other serious side effects. In a study conducted by Browne et al. ${ }^{6}, 10.0 \%$ of patients knew that sulfonylurea derivatives could cause hypoglycemia. An increased hypoglycemia awareness as well as overall knowledge about diabetes therapy among patients may be important determinants in the management of type 2 DM. $^{8}$

It is well-recognized that the treatment of patients with type $2 \mathrm{DM}$ is associated with the use of multiple medications, which include not only glucose-lowering therapies but also treatments for accompanying disorders. Poor knowledge concerning side effects in these patients is not unexpected due to the amount of information they need to know about the other drugs they take as well. Multiple medications are also associated with more adverse events as well as drug interactions. ${ }^{9}$ Our research has shown that more than half of the respondents $(56.0 \%)$, besides OHAs, used another 1-3 drugs for the treatment of other health disorders. Indeed, several studies have suggested that a patient with type 2 DM can often expect to take as many as five or more medications daily. ${ }^{10,11}$

In addition to their poor knowledge on OHA side effects, $30.0 \%$ of respondents gave inaccurate answers when asked what they would do if they noticed a side effect. It is interesting to note that only $1.0 \%$ of them responded they would consult a pharmacist, which was deemed the correct answer, indicating the need for discussion regarding the patients' reliance on pharmacists as well as their role in educating patients while dispensing drugs at a pharmacy. Due to the trend of the recent years, at present, most of the pharmacies in Serbia are privately owned, and they focus mainly on sales rather than giving information to patients. Furthermore, this study's participants maintained they had obtained information about $\mathrm{OHAs}$ by endocrinologists the most, followed by general practitioners, while a single respondent $(1.0 \%)$ stated the pharmacist as the primary source of information.

When it comes to the practical application of their knowledge regarding $\mathrm{OHAs}$, it was found that $38.0 \%$ of patients did not know correct way to proceed with the treatment after missing a dose of their drug, indicating that they were not sufficiently informed about this issue. It is important to note that although $99.0 \%$ of patients claimed to know at what time of day and how many times a day they should take the drug, only $7.0 \%$ of them answered that they had never missed taking a dose, which indicates on a poor adherence to treatment. Several medicationrelated factors can influence treatment adherence in patients with type 2 DM. Overall, regimen complexity, including dosing frequency, impairs adherence. Patient understanding of the drug's dosing regimen and its therapeutic value are important for good adherence. Other factors tend to impair adherence are depression and forgetfulness. However, failure to remember to take one's medications is not solely a feature of affective disorders. In a study of medication usage in a real-world, cross-sectional population of patients with type 2 DM treated for $\geq 10$ years, $20.0 \%$ of participants reported forgetting to take their medications regularly and without any particular explanation. ${ }^{12}$ Medication adherence in patients with type $2 \mathrm{DM}$ is a topic of great interest because good adherence in these patients is one of the most important factors for maintaining normal blood glucose levels and, hence, preventing severe complications.

In a study conducted in Northern Ireland by Williams and colleagues ${ }^{13}$, patients were also scored on their $\mathrm{OHA}$ knowledge using a scale of 0-8 after answering similar 
questions. There was a difference in two questions between the two studies, however-instead of our questions "What would you do if you missed a dose?" and "What would you do if you noticed a side effect?", they asked the patients to recall the color and shape of the OHA tablets). Most of the patients in their study achieved a score of 3-5 (44.0\%). When asked who gave them the greatest quantity of information about OHAs, diabetes specialist nurses $(52.2 \%)$ and general practitioners (28.3\%). were the most commonly cited health professionals. Moreover, two thirds (64.6\%) of patients felt they had been given enough information about OHAs. In addition, a study conducted by Browne and colleagues ${ }^{6}$ found a better level of patient knowledge regarding the OHAs' mechanism of action compared to our study. They reported that $15.0 \%$ of their patients knew the correct mechanism of action of OHAs. Different from our study, the pharmacist was rated as the main information provider by $38.8 \%$ of patients with type $2 \mathrm{DM}$ in research conducted by Ojieabu and colleagues in Nigeria. ${ }^{14}$ A study conducted in rural India showed that only $42.5 \%$ of patients knew the names of their OHAs, 53.2\% knew the indication for their use, $48.9 \%$ understood their dosing frequency and directions for their use, while $31.9 \%$ of patients could recall one side effect. ${ }^{15}$

Norris et al. ${ }^{16}$ evaluated the efficacy of self-management education on lowering/controlling glycated hemoglobin levels in adults with type 2 diabetes and concluded that short-term education improves glycated hemoglobin levels at the immediate follow-up. Still, the benefit declines 1-3 months after the intervention ceases. Moreover, longer follow-ups monitoring the effects of diabetes education have shown that it is effective in the long term. ${ }^{17}$ Izquierdo et al. ${ }^{18}$ recommend that diabetes education be provided as an effective program via new technology such as telemedicine.

Our study had certain limitations that need to be mentioned. The first limitation is its relatively small sample of respondents. Secondly, the study was conducted in one city; therefore, its data may not represent the actual situation regarding this topic in the whole country.

\section{Conclusion}

Although our patients with type 2 DM achieved good scores regarding knowledge about OHAs, more than half of them still perceived that their knowledge about these drugs was insufficient. The results clearly indicate that this patient sample was not sufficiently familiar with the mechanism of action of these drugs and their possible side effects. Information about OHAs given in written form at the time their initial prescription by the physician, as well as oral explanations by pharmacists at each drug dispensing session, would contribute significantly to the education of type 2 diabetes patients about $\mathrm{OHAs}$, and as a consequence, to a better management of their condition.

\section{Funding sources}

This research was supported by the Ministry of Education, Science and Technological Development, Republic of Serbia, project No. 41012.

\section{Conflicts of interest}

Nothing to declare.

\section{References}

1. NCD Risk Factor Collaboration (NCD-RisC). Worldwide trends in diabetes since 1980: a pooled analysis of 751 populationbased studies with 4.4 million participants. Lancet 2016;387: 1513-30.

2. Zheng Y, Ley SH, Hu FB. Global aetiology and epidemiology of type 2 diabetes mellitus and its complications. Nat Rev Endocrinol 2018;14:88-98.

3. Brennan GM, Boyle DIR, McMahon AD, MacDonald TM, Jung RT, Newton RW, et al. Do patients with diabetes take their tablets? Diabetic Med 1998;15:S17. 
4. Dunn CJ, Peters DH. Metformin. A review of its pharmacological properties and therapeutic use in non-insulin dependent diabetes mellitus. Drugs 1995;49:721-49.

5. Vermeire E, Van Royen P, Coenen S, Wens J, Denekens J. The adherence of type 2 diabetes patients to their therapeutic regimens: a qualitative study from the patient's perspective. Practical Diabetes Int 2003;20:209-14.

6. Browne DL, Avery L, Turner BC, Kerr D, Cavan DA. What do patients with diabetes know about their tablets? Diabetic Med 2000;17:528-31.

7. Marks L. Counting the cost: the real impact of non-insulin dependent diabetes. London: British Diabetic Association; 1996.

8. Sarkar U, Karter AJ, Liu JY, Adler NE, Nguyen R, López A, et al. The literacy divide: health literacy and the use of an internetbased patient portal in an integrated health system-results from the diabetes study of northern California (DISTANCE). $\mathrm{J}$ Health Commun 2010;15:183-96.

9. Austin RP. Polypharmacy as a risk factor in the treatment of type 2 diabetes. Diabetes Spectr 2006;19:13-6.

10. Ibrahim IA, Kang E, Dansky KH. Polypharmacy and possible drug-drug interactions among diabetic patients receiving home health care services. Home Health Care Serv Q 2005;24: 87-99.

11. Dunning T, Manias E. Medication knowledge and self- management by people with type 2 diabetes. Aust $\mathrm{J}$ Adv Nurs 2005;23:7-14.

12. Bailey CJ, Kodack M. Patients adherence to medication requirements for therapy of type 2 diabetes. Int $\mathrm{J}$ Clin Pract 2011;65:314-22.

13. Williams L, Caskey H, Coates V, Thompson K, Stewart H. A survey of patients' knowledge on their diabetes medication. J Diabetes Nurs 2007;11:264-9.

14. Ojieabu WA, Arute JE, Ajayi TD. Assessment of medication knowledge among adults with diabetes mellitus in a Nigerian Teaching Hospital. Annals of Health Research 2015;1:62-7.

15. Gangwar SS, Ahmad A, Patel I, Parimalakrishnan S, Balkrishnan R, Singh SP. Assessment of medication knowledge and counseling in type 2 diabetes mellitus patients attending community pharmacy in rural north India. AJPSP 2013;4:4757.

16. Norris SL, Lau J, Smith J, Schmid CH, Engelgau MM. SelfManagement education for adults with type 2 diabetes. Diabetes Care 2002;25:1159-71.

17. Thongsai S, Youjaiyen M. The long-term impact of education on diabetes for older people: a systematic review. Glob J Health Sci 2013;5:30-9.

18. Izquierdo RE, Knudson PE, Meyer S, Kearns J, Ploutz-Snyder R, Weinstock RS. A comparison of diabetes education administered through telemedicine versus in person. Diabetes Care 2003;26:1002-7. 